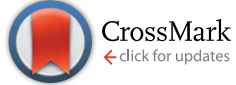

Cite this: RSC Adv., 2017, 7, 3676

Received 14th November 2016 Accepted 23rd December 2016

DOI: 10.1039/c6ra26791b

www.rsc.org/advances

\section{Cost effective surface functionalization of gold nanoparticles with a mixed DNA and PEG monolayer for nanotechnology applications $\uparrow$}

\author{
Risheng Wang, ${ }^{\text {a }}$ Isabella Bowling ${ }^{a}$ and Wenyan Liu ${ }^{b}$
}

A cost effective and generally applicable co-functionalization of gold nanoparticles with a mixed DNA and PEG polymers is reported. Compared to the pure DNA functionalization method, this protocol uses a much smaller amount of thiol-DNA to achieve a similar binding efficacy of gold nanoparticles with DNA origami nanostructures. The lower consumption of DNA provides a scaling-up potential for the application of DNAnanoparticle conjugates in nanotechnology.
With the rapid development of nanotechnology, biomolecule conjugated nanoparticles have been playing more central roles in recent applications. ${ }^{1-4}$ In particular, DNA functionalized gold nanoparticles (DNA-GNPs), combined with the sequencedependent recognition properties of DNA and the unique electronic, optical, and catalytic properties of GNPs, have greatly stimulated widespread interest in various fields, including biosensing, ${ }^{5,6}$ drug delivery, ${ }^{7}$ imaging, ${ }^{8,9}$ and catalysis. ${ }^{10,11}$ Studies have shown that the stability of DNA-GNP conjugates has a significant impact on these applications. ${ }^{12} \mathrm{~A}$ salting-aging thiol-DNA/GNP conjugation procedure, based on the high affinity between thiol and gold, is the most commonly applied method for achieving stable DNA-GNP conjugates. ${ }^{13}$ However, this approach requires an excessive number of DNA strands to stabilize GNPs against salt-induced aggregation, which leads to a limited number of the DNA strands being attached on the particle surface and a majority of the unconjugated thiol-DNA being discarded. ${ }^{14}$ While the chemical synthesis of DNA has been well developed, ${ }^{15}$ the cost of thiol-DNA is still a major obstacle to GNP functionalization by using large amount of DNA. Thus, this method dramatically limits the scale-up of the DNA-GNP hybrids and their integration with lithography or other techniques that require large amounts of materials. ${ }^{16}$ Therefore, it is highly desirable to develop a strategy that can reduce the consumption of DNA strands during functionalization, while still maintaining the stability and bio-functionality of DNA-GNP hybrids.

Co-functionalization of GNPs with DNA and other types of ligands is a possible route for reducing the costs required to

${ }^{a}$ Department of Chemistry, Missouri University of Science and Technology, Rolla, MO 65409, USA.E-mail: wangri@mst.edu

${ }^{b}$ Environmental Research Center, Missouri University of Science and Technology, Rolla, MO 65409, USA

$\dagger$ Electronic supplementary information (ESI) available. See DOI: 10.1039/c6ra26791b scale-up the production of DNA-GNPs conjugates. To date, however, several ligands such as polyethylene glycol (PEG), ${ }^{17-19}$ peptides, ${ }^{20,21}$ and polyvinylpyrrolidone (PVP), ${ }^{22}$ have been reported to decorate GNPs with DNA. Unfortunately, those studies only focused on using ligands to improve the nanoparticles' stability, ${ }^{20,22}$ cellular internalizations, ${ }^{20}$ nuclease degradation resistance, ${ }^{18}$ and DNA conjugation speed. ${ }^{23}$ They lacked sufficient information about whether or not the mixed monolayer affected the bio-functionality of co-functionalized GNPs. Importantly, there is no discussion about the possibility of functionalizing GNPs with a minimum amount of DNA, while still keeping the DNA binding efficiency similar to that of sole DNA decorated GNPs.

In this work, we experimentally investigated whether DNAGNP conjugates, with excellent stability and bio-functionality, can be achieved through co-immobilizing PEG on particle surfaces to reduce the amount of DNA used. This study was carried out by functionalizing GNPs with various proportions of mixed thiol-terminated DNA/PEG monolayers. The biofunctional behaviour of the functionalized GNPs was then tested by hybridization with DNA origami nanostructures ${ }^{24}$ via sequence-complementarity. Our results showed that the DNA/ PEG co-immobilized GNPs, but still maintained excellent biofunctionality. As little as an eighth of the typical amount of DNA was used during functionalization, as compared with the amount required for the traditional method. This study will open the door for the scale-up production of DNA conjugated GNPs through more efficient utilization of chemically synthesized thiol-DNA.

To explore the possibility of utilization of PEG as a co-ligand to reduce the amount of DNA needed during GNP functionalization, a two-step functionalization procedure was adopted (shown in Fig. 1). First, the surfaces of $15 \mathrm{~nm}$ GNPs were coated with a certain amount of thiol-DNA strands, via a salting-aging process. Second, the resulting particles were back filled with 

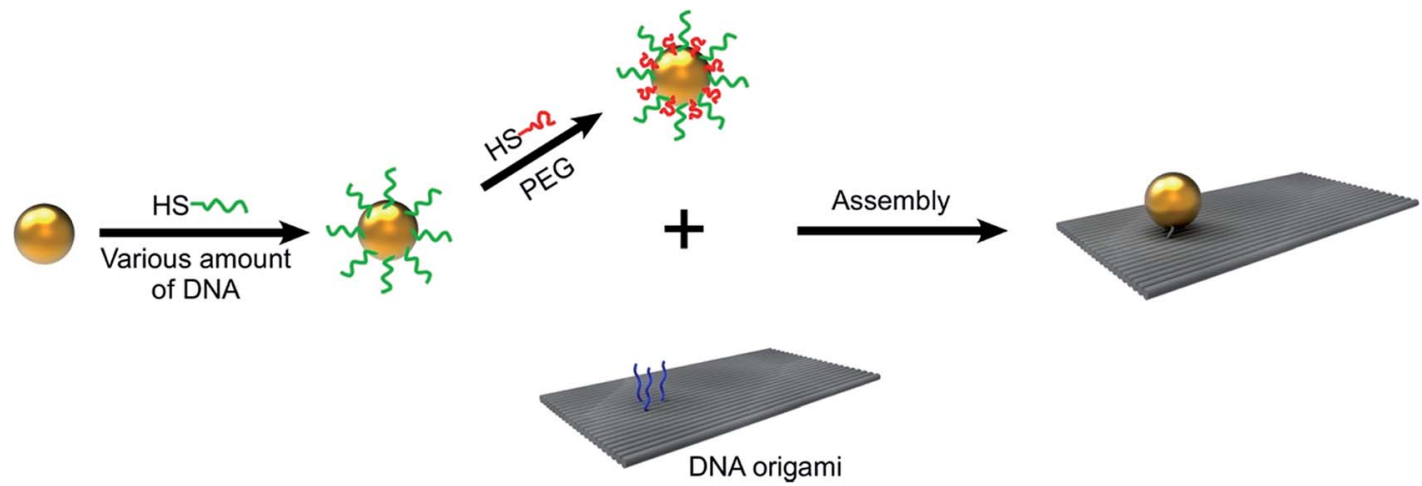

Fig. 1 Schematic illustration of two-step functionalization of GNPs, with DNA/PEG polymers containing varied amounts of DNA and their attachment with rectangular DNA origami.

thiol terminated PEG polymers to improve their stability, followed by removal of the unconjugated DNA and PEG, via centrifugation. It has been proven that a higher density of PEG on a gold surface limits the following step conjugation of DNA. In addition, if PEG is longer than DNA, it interferes with DNA hybridization efficacy on particle surfaces. ${ }^{19}$ Therefore, based on this knowledge, a $3^{\prime}$ thiol-terminated 20-mer thymine sequence of DNA (length: $\sim 6.5 \mathrm{~nm}$ ) was chosen to modify GNPs, and a low-molecular-weight PEG (MW 356 Da, length: $\sim 2.4 \mathrm{~nm}$ ), shorter than the thiol-DNA, was used for backfilling. To make a quantitative comparison, four types of GNPs were prepared: $\mathrm{GNP}_{800}, \mathrm{GNP}_{400 \mathrm{p}}, \mathrm{GNP}_{200 \mathrm{p}}$, and $\mathrm{GNP}_{100 \mathrm{p}}$ (numbers in subscript represent the molar ratio between DNA and GNPs, and "p" stands for PEG backfilling). For example, GNPs functionalized with a $800: 1$ DNA to GNP ratio were named as $\mathrm{GNP}_{800}$, Also, GNPs functionalized with a $200: 1$ DNA to GNP ratio and those backfilled by PEG were named $\mathrm{GNP}_{200 \mathrm{p}}$. It should be noted that for two particles, $\mathrm{GNP}_{200 \mathrm{p}}$, and $\mathrm{GNP}_{100 \mathrm{p}}, 0.01 \%$ sodium dodecylsulfate (SDS) was added to the conjugation mixture during the first step to avoid particle aggregation. Also, the unconjugated DNA was not removed after the first salt-aging process for two reasons: first, to simplify the experimental procedure, and second, to increase DNA conjugation yield in order to pursue the maximum bio-functionality of particles with a limited source of DNA.

To systematically evaluate the quality and functionality of the co-functionalized GNPs, binding tests were performed by DNA hybridization between an anchoring site on planar rectangular DNA origami templates (see ESI $\dagger$ for detailed design) and the complementary single-stranded DNA (ssDNA) coating on the GNPs. The anchoring site was composed of three 25-mer single-stranded poly (A) extensions protruding out from the DNA origami surface. Previous to hybridization, the DNA origami nanostructures were annealed and purified to remove excess staple strands using the protocol adopted in our previous publication..$^{25}$ These origami nanostructures were then mixed with the co-functionalized GNPs at a ratio of $1: 3$, and followed by slow cooling for 12 hours at temperatures ranging from $47^{\circ} \mathrm{C}$ to room temperature. Atomic force microscopy (AFM) revealed the binding efficiency between the GNPs and the DNA origami templates (Fig. 2).
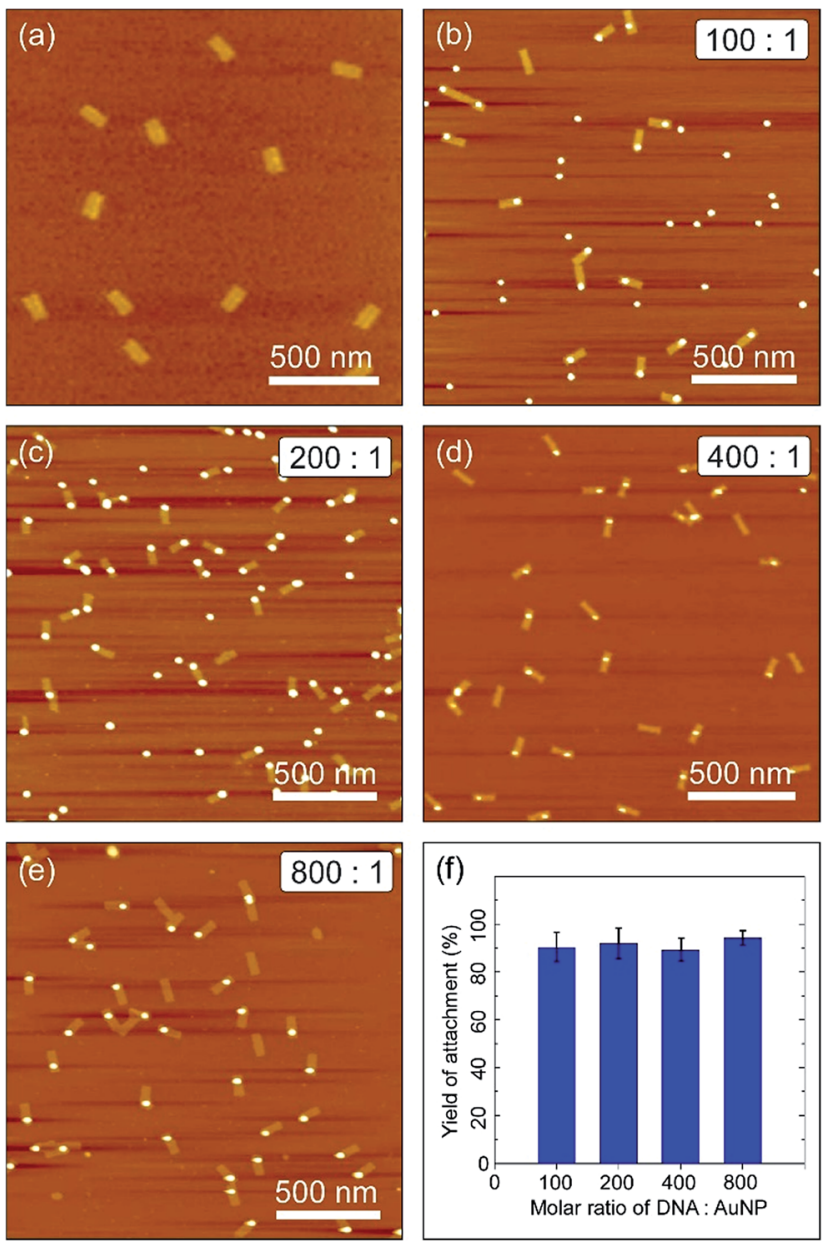

Fig. 2 The attachment yield of co-functionalized GNPs with a rectangular DNA origami template. (a) AFM image of the rectangular DNA template. (b-e) AFM images of GNP/DNA origami assemblies with $\mathrm{GNP}_{100 p}, \mathrm{GNP}_{200 p}, \mathrm{GNP}_{400 p}, \mathrm{GNP}_{800}$, respectively. (f) The hybridization yield is $90.2 \%$ (total number of DNA origami $N=591$ from $n=11$ independent measurements) for (b), $91.9 \%(N=607, n=12)$ for (c), $89.1 \%(N=960, n=10)$ for $(d)$, and $94.2 \%(N=624, n=8)$ for $(\mathrm{e})$. The AFM images were scanned with a tapping mode in air. 
As can be seen in Fig. 2a, the origami templates were formed as designed in high yield with well-defined shapes and sizes. Fig. 2b-e show AFM images of the assembly of DNA/PEG-GNPs on the rectangular DNA origami platforms. It is clear from these images that the two components in all four cases $\left(\mathrm{GNP}_{800}\right.$, $\mathrm{GNP}_{400 \mathrm{p}}, \mathrm{GNP}_{200 \mathrm{p}}$, and $\mathrm{GNP}_{100 \mathrm{p}}$ ) hybridized together in a $1: 1$ ratio. The yield analysis based on the AFM images, as shown in the histogram in Fig. 2f, reveals that their binding efficiencies were very close to each other, and the binding yield of the DNA/ PEG-GNP hybrids still remained high. For example, the binding yields of $\mathrm{GNP}_{200 \mathrm{p}}$ and $\mathrm{GNP}_{100 \mathrm{p}}$ were $91.9 \%$ and $90.2 \%$, respectively, which is comparable to the binding yield of sole DNA functionalized GNPs (94.2\%). This indicated that the DNA/PEG co-functionalized GNPs had excellent hybridization capability which was similar to that of sole DNA coated GNPs, even at very low DNA surface coverage.

Subsequently, the DNA/PEG-GNPs, and their conjugation with DNA origami templates, were analysed by agarose gel electrophoresis. The gel images under UV-light and white light are shown in Fig. 3a and b, respectively. Lane 1 corresponds to the rectangular DNA origami as a reference. Lanes $2,4,6$, and 8 represent DNA/PEG-GNPs $\left(\mathrm{GNP}_{800}, \mathrm{GNP}_{400 \mathrm{p}}, \mathrm{GNP}_{200 \mathrm{p}}\right.$, and $\mathrm{GNP}_{100 \mathrm{p}}$, respectively), while lanes $3,5,7,9$ represent the DNA origami and GNP complexes in which the GNPs correspond to those in lane 2, 4, 6 and 8, respectively. The slightly decreased mobility of the complexes, compared with the DNA origami, was due to the attachment of nanoparticles. As can be seen, each complex appeared as a single and clear band with similar intensity, indicating that each GNPs had a similar binding affinity to the DNA origami, which was consistent with statistical analysis data obtained from AFM.

The optical properties of GNPs were also an interesting feature for potential applications. It is especially necessary to characterize GNPs after surface functionalization with biomolecules. The UV-vis spectrum was measured for GNPs and DNA/PEG-decorated GNPs, as shown in Fig. 4. The typical

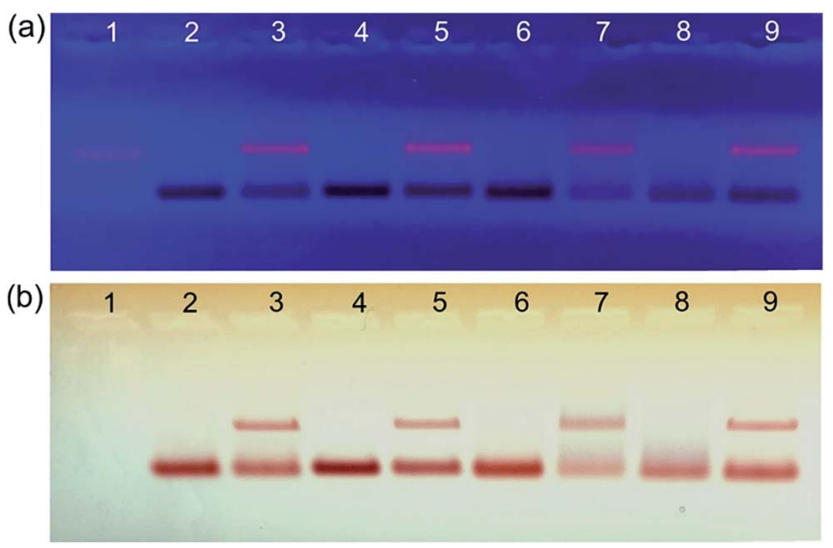

Fig. 3 Ethidium bromide-stained agarose gel electrophoretic images of the GNP/DNA origami assemblies under UV light (a) and daylight (b). Lane 1: DNA origami. Lane 2, 4, 6, 8: DNA/PEG functionalized GNPs. The ratio of DNA : GNPs is $800: 1,400: 1,200: 1,100: 1$ respectively. Lane 3, 5, 7, 9: GNP/DNA origami assemblies. The GNPs are corresponding to lanes $2,4,6,8$, respectively.

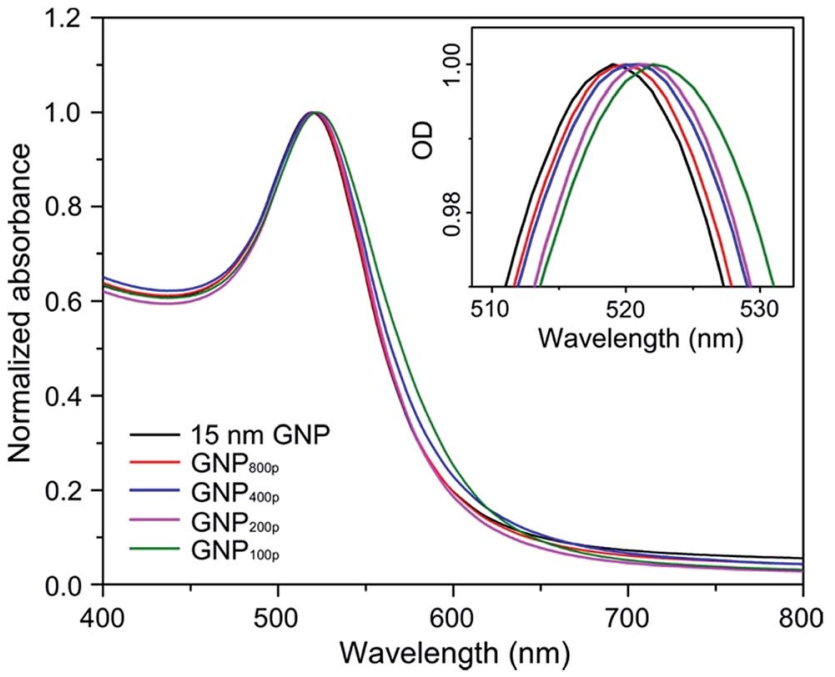

Fig. 4 UV-vis absorbance spectra of unmodified GNPs and DNA/PEG functionalized GNPs. The inset shows a magnification of the absorbance peak.

plasmon band of $15 \mathrm{~nm}$ GNPs in an aqueous solution showed absorption maxima of around $520 \mathrm{~nm}$. After DNA/PEG conjugation, a small red shift of the plasmon absorption band was observed (inset of Fig. 4), indicating the surface modification. The extent of shift increased with an increasing amount of PEG because of changes in the dielectric constant of the GNP surface. The position of the plasmon band was extremely sensitive to size, shape, agglomeration state, and surrounding environment. ${ }^{26}$ Fig. 4 shows that the conjugation of DNA and PEG to the GNP surface did not cause significant changes in the shape and position of the plasmon bands. This indicated that the co-functionalization method did not change a GNP's optical properties, ${ }^{26}$ which is critical for their applications in the optical biosensor and biomedical imaging system.

In summary, we have demonstrated a cost-effective method to functionalize GNPs with mixed DNA/PEG polymers. We systematically decreased the amount of DNA utilization and increased the amount of PEG attachment to the surfaces of particles. The bio-functionality of particles that were prepared following this protocol was ensured by conjugated DNA, and its stability was attained by PEG passivation. Compared to the pure DNA functionalization method, this protocol used a much smaller amount of DNA to achieve a similar binding efficacy of GNPs with DNA nanostructures, though we didn't explore the minimum limitation. We also demonstrated that a coated DNA/ PEG monolayer on GNP surfaces did not affect the optical properties of particles. This robust protocol can be extended from spherical particles to anisotropic particles, since their functionalization will consume more DNA with increased surface areas and more complicated crystal features. The lower consumption of DNA, in turn, provides a scaling-up potential for the application of DNA-GNP conjugates in optical, biomedical, nanoengineering, and more advanced applications of DNA nanotechnology. 


\section{Acknowledgements}

We gratefully acknowledge financial support from the University of Missouri Research Board, the Center for Biomedical Science and Engineering at Missouri S \& T, and the Material Research Center for equipment usage. The authors would also like to thank Dr Switzer for helpful discussions.

\section{Notes and references}

1 C. Zhang, R. J. Macfarlane, K. L. Young, C. H. Choi, L. Hao, E. Auyeung, G. Liu, X. Zhou and C. A. Mirkin, Nat. Mater., 2013, 12, 741-746.

2 R. Baron, B. Willner and I. Willner, Chem. Commun., 2007, 323-332.

3 Y. Zhang, R. Huang, X. Zhu, L. Wang and C. Wu, Chin. Sci. Bull., 2012, 57, 238-246.

4 C. C. Wang, S. M. Wu, H. W. Li and H. T. Chang, ChemBioChem, 2016, 17, 1052-1062.

5 J. Liu and Y. Lu, Angew. Chem., 2005, 45, 90-94.

6 M. Oishi and S. Sugiyama, Small, 2016, 12, 5153-5158.

7 S. Dhar, W. L. Daniel, D. A. Giljohann, C. A. Mirkin and S. J. Lippard, J. Am. Chem. Soc., 2009, 131, 14652-14653.

8 D. S. Seferos, D. A. Giljohann, H. D. Hill, A. E. Prigodich and C. A. Mirkin, J. Am. Chem. Soc., 2007, 129, 15477-15479.

9 C. Wang, J. Zhou, P. Wang, W. He and H. Duan, Bioconjugate Chem., 2016, 27, 815-823.

10 D. Yao, H. Li, Y. Guo, X. Zhou, S. Xiao and H. Liang, Chem. Commun., 2016, 52, 7556-7559.

11 P. Zhou, S. Jia, D. Pan, L. Wang, J. Gao, J. Lu, J. Shi, Z. Tang and H. Liu, Sci. Rep., 2015, 5, 14402.

12 F. Li, H. Zhang, B. Dever, X. F. Li and X. C. Le, Bioconjugate Chem., 2013, 24, 1790-1797.
13 W. Zhao, L. Lin and I. M. Hsing, Bioconjugate Chem., 2009, 20, 1218-1222.

14 X. Zhang, M. R. Servos and J. Liu, J. Am. Chem. Soc., 2012, 134, 7266-7269.

15 M. H. Caruthers, J. Chem. Educ., 1989, 66, 577-580.

16 A. M. Hung, C. M. Micheel, L. D. Bozano, L. W. Osterbur, G. M. Wallraff and J. N. Cha, Nat. Nanotechnol., 2010, 5, 121-126.

17 K. Yoshimoto, S. Matsumoto, R. Asakawa, K. Uchida, T. Ishii and Y. Nagasaki, Chem. Lett., 2007, 36, 1444-1445.

18 L. Song, Y. Guo, D. Roebuck, C. Chen, M. Yang, Z. Yang, S. Sreedharan, C. Glover, J. A. Thomas, D. Liu, S. Guo, R. Chen and D. Zhou, ACS Appl. Mater. Interfaces, 2015, 7, 18707-18716.

19 A. Takashima and M. Oishi, RSC Adv., 2015, 5, 76014-76018. 20 E. Harrison, J. R. Nicol, M. Macias-Montero, G. A. Burke, J. A. Coulter, B. J. Meenan and D. Dixon, Mater. Sci. Eng., C, 2016, 62, 710-718.

21 S. Rana, A. Bajaj, R. Mout and V. M. Rotello, Adv. Drug Delivery Rev., 2012, 64, 200-216.

22 J. H. Heo, K. I. Kim, H. H. Cho, J. W. Lee, B. S. Lee, S. Yoon, K. J. Park, S. Lee, J. Kim, D. Whang and J. H. Lee, Langmuir, 2015, 31, 13773-13782.

23 J. Li, B. Zhu, Z. Zhu, Y. Zhang, X. Yao, S. Tu, R. Liu, S. Jia and C. J. Yang, Langmuir, 2015, 31, 7869-7876.

24 F. N. Gur, F. W. Schwarz, J. Ye, S. Diez and T. L. Schmidt, ACS Nano, 2016, 10, 5374-5382.

25 R. Wang, C. Nuckolls and S. J. Wind, Angew. Chem., 2012, 51, 11325-11327.

26 L. Rastogi, A. J. Kora and A. Jayaraman, Mater. Sci. Eng., C, 2012, 32, 1571-1577. 